\title{
BMJ Open Models of support to family members during the trajectory of cancer: a scoping review protocol
}

\author{
Maria Samuelsson (D) , ${ }^{1}$ Anne Wennick, ${ }^{2}$ Jenny Jakobsson, ${ }^{1}$ Mariette Bengtsson ${ }^{3}$
}

\begin{abstract}
To cite: Samuelsson M, Wennick A, Jakobsson J, et al. Models of support to family members during the trajectory of cancer: a scoping review protocol. BMJ Open 2020;10:e037633. doi:10.1136/ bmjopen-2020-037633
\end{abstract}

- Prepublication history for this paper is available online. To view these files, please visit the journal online (http://dx.doi org/10.1136/bmjopen-2020037633).

Received 10 February 2020 Revised 23 June 2020 Accepted 30 July 2020

A) Check for updates

(c) Author(s) (or their employer(s)) 2020. Re-use permitted under CC BY-NC. No commercial re-use. See rights and permissions. Published by BMJ.

${ }^{1}$ Department of Care Science, Malmö Universitet, Malmö, Skåne, Sweden

${ }^{2}$ Faculty of Health and Society, Care Science, Malmö University, Malmö, Skåne, Sweden

${ }^{3}$ Care Science, Malmö University, Malmö, Sweden

Correspondence to

Maria Samuelsson;

maria.samuelsson@mau.se

\begin{abstract}
Introduction A cancer diagnose, for example, colorectal cancer, not only affects the cancer-person stricken, but also the surrounding family. Thus, this scoping review intends to identify appropriate models of support that will guide the development of a model of support to family members during the trajectory of colorectal cancer. Methods and analysis This scoping review will be guided by the methodological framework developed by Arksey and O'Malley, refined by Levac et al and Colquhoun et al, and described by the Joanna Briggs Institute. All the stages will be conducted iteratively and reflexively. First, a search strategy will be developed with a librarian and applied in the following peer-reviewed databases: PubMed, Cumulative Index to Nursing and Allied Health Literature and PsycINF0. Additional searches will be performed in Google Scholar and SwePub for identification of grey literature and hand searched in the reference lists. Searches will be conducted from December 2019 to February 2020. A draft of the preliminary search strategy was performed in PubMed in November 2019. Subsequently, three members of the research team will independently screen all abstracts for relevance, as well as the full-text articles. Studies meeting the inclusion criteria will be critically evaluated using the Joanna Brigg Institute Critical Appraisal Tools. A descriptive summary of study characteristics and of the scoping review process will be presented, including a visual flow diagram. Lastly, a thematic analysis as presented by Braun and Clarke will be conducted. To enhance validity, contact nurses of persons with colorectal cancer will be provided an overview of the preliminary results.
\end{abstract}

Ethics and dissemination Being a secondary analysis, ethical approval is not needed for this study. The findings of the analysis will be used to inform the design of a future study aiming to develop a model of support and an upcoming scoping review, which will be published in a scientific journal and presented at relevant conferences.

\section{INTRODUCTION}

Throughout the trajectory of cancer-from diagnose, through treatment and on to survivorship or palliative care-family members are described as the most important means of support. ${ }^{12}$ Though the provided support is important, it is not unproblematic. It is well known that family members play an important role in the stricken person's compliance with

\section{Strengths and limitations of this study}

- The scoping review will enable identification of appropriate models of support to family members during the trajectory of cancer, which will guide the development of a model of support and future studies.

- Search strategies will be developed in collaboration with a research librarian well versed in using research databases and in developing search strategies.

- The search strategy will include three different databases with peer-reviewed literature, with no restrictions in study design or publication year and with an additional search of grey literature.

- Only literature in English and Swedish will be included.

treatment regimens and activities in her or his everyday life, ${ }^{34}$ or even that being married or in a similar relationship has a positive impact on the person's survival. ${ }^{3}$ Nevertheless, family members of persons diagnosed with cancer themselves are at risk of becoming ill. $^{3-5}$ Family members show higher rates of anxiety, depression and weakened immune response, ${ }^{3}$ reactions to severe stress and ischaemic heart disease. ${ }^{5}$ In addition, being a family member to a person with a cancer diagnose means an increased likelihood of long-term medical problems and higher mortality. ${ }^{4}$ Likewise, family members of persons diagnosed with colorectal cancer describe how they become responsible for not only the stricken person's well-being, but also compliance with hers or his everyday life-a responsibility experienced as a heavy burden. ${ }^{12}$ Moreover, the treatment and recovery process from colorectal cancer is described as having a severe impact on family members. ${ }^{1}$ Furthermore, an illness, for example, colorectal cancer, not only affects the family members, but also the relationships within the family, and it challenges established communication patterns, roles and responsibilities. ${ }^{6}$ Thus, colorectal cancer is an illness that may affect the family system 
itself. For this reason, health professionals must be aware of the possible needs of family members; and even if they demonstrate a range of strengths, they are vulnerable during this stressful period. ${ }^{7}$ Consequently, there is a necessity not only to address the needs of support of the ill person's partner, but also the needs of other family members.

In short, even though support resources exist within a family, there is no guarantee such potential support can overcome the negative impact colorectal cancer may have on both the family members and on the family system. This means that without adequate support, family members themselves are at risk of becoming ill; thus, the most prominent resource of support may be lost. Despite the severe impact colorectal cancer has on family members, the support offered by healthcare professionals is experienced as entirely patient focused. ${ }^{16}$ Therefore, this scoping review will be conducted to address the apparent need to focus on the family members' needs of support. In addition, as no support model was found focusing on the families of persons diagnosed with colorectal cancer, the review will scope the literature on support provided to family members during the trajectory of all cancers. Thus, the scoping review intents not only to identify appropriate models of support, but also to identify gaps in knowledge regarding, for example, phases of the trajectory. The results will guide the development of a model of support to family members during the trajectory of colorectal cancer care and the design of further studies. In preparation for this scoping review, searches were made to locate a comparable, published or on-going, systematic and/or scoping review in PubMed, Cumulative Index to Nursing and Allied Health Literature (CINAHL), Cochrane Library and PROSPERO. However, none were identified.

\section{AIM}

The aim of the scoping review is to map the existing literature on models of support provided to family members during the trajectory of cancer.

\section{METHODS AND ANALYSIS}

The study will be conducted as a scoping review of the existing literature on models of support provided to family members during the trajectory of cancer. A scoping review was chosen as it, according to Levac et $a l^{8}$ facilitates the mapping of new concepts, types of evidence and gaps of knowledge. To ensure rigour in methodology reporting, the present study will follow the six-stage approach developed by Arksey and O'Malley, ${ }^{9}$ refined by Levac et $a l^{8}$ and Colquhoun $e t a l,{ }^{10}$ and described by the Joanna Briggs Institute ${ }^{11}$ : (1) identifying the research question, (2) identifying relevant studies, (3) selecting studies, (4) charting the data, (5) collating, summarising and reporting the results, (6) consultation. Reporting will be compliant with the Preferred Reporting Items
Table 1 The Population, Concept and Context mnemonic as recommended by Joanna Briggs Institute

$\begin{array}{ll}\text { Participants } & \begin{array}{l}\text { Family members of a person diagnosed with } \\ \text { cancer. }\end{array} \\ \text { Concept } & \text { Models of support. } \\ \text { Context } & \begin{array}{l}\text { The trajectory of cancer. Both in hospital and } \\ \text { home setting. }\end{array}\end{array}$

for Systematic Reviews and Meta-Analyses Extension for Scoping Reviews Checklist ${ }^{12}$ (PRISMA-ScR).

\section{Stage 1: identifying the research question}

The research question for this scoping review is as follows: What is known from the existing literature about models of support provided to family members during the trajectory of cancer? In line with the recommendations by Arksey and $\mathrm{O}^{\prime}$ Malley, ${ }^{9}$ the research question was formulated to generate breadth of coverage by maintaining a wide approach. Since scoping is an iterative process, additional questions may be added based on our findings along the review process.

The initial specific research questions of this scoping review are the following: (1) What are the characteristics of the models described? (2) During which phase of the trajectory is the described support provided? (3) What are the aims of the support? (4) To whom is the support directed? (to multiple family members or to which family member?)

\section{Stage 2: identify relevant studies}

The initial search protocol was designed by the research team and developed in collaboration with a research librarian well versed using research databases. The scoping review will use the mnemonic Population, Concept and Context, described by Joanna Briggs Institute ${ }^{11}$ (table 1) to establish effective search criteria.

To get a wide-ranging picture of the existing research, studies of different designs will be included, that is, qualitative, quantitative and mixed method design, to address the research questions. The search strategy will be conducted iteratively by the research team, which means the researcher being reflexive at all steps and, when necessary, repeating steps to ensure that the literature is covered in a comprehensive way. ${ }^{9}$ Electronic searches will be conducted in the following peer-reviewed databases: PubMed, CINAHL and PsycINFO. Search tools such as Medical Subject Headings, Headings, Thesaurus and Boolean operators (AND/OR) will be used to expand and narrow the search and keywords, for example, support, neoplasm and synonyms of for example, family, next of kin, partner, nuanced to apply to the different databases. No limitations will be set to the year of publication. Finally, search strategies will be developed in collaboration with a research librarian. A draft search of the preliminary search strategy was conducted in PubMed in 20191114 (online supplemental file). In accordance with the Johanna Briggs Institute, ${ }^{11}$ the process will start 
with a broad search to inform the subsequent. Words contained in the title and abstracts, and the index terms describing the papers, will be analysed by two members of the research team and the search strategy refined before conducted in all databases. As a third step in the search strategy, the reference lists of retrieved articles will be hand searched for additional studies. Searches will also be conducted using Google Scholar and SwePub to identify grey literature.

\section{Inclusion and exclusion criteria}

Types of articles to be considered for inclusion are articles that present (a) primary research about support provided by healthcare (b) to family members (c) during the trajectory of cancer (d) of an adult person (e) in Swedish or English (f) studies evaluated with moderate or high methodological quality. The references in the grey literature will be searched to identify unpublished studies. The scoping review will exclude books, book reviews, commentaries, letters to the editor and abstracts for conferences, as the interest pertains to models of support described in original research. Reviews will be excluded, but their reference list will be hand searched. The inclusion and exclusion criteria may be modified within the iterative, dynamic process, resulting in revisiting and refining the search strategy, as described by Colquhoun et al. ${ }^{10}$ If so, the process will be described in the final manuscript.

\section{Stage 3: study selection}

All the titles from the second search will be screened for relevance by one member of the research team. If the title is in line with the aim of the study, the abstracts will be read and independently assessed with the eligibility criteria by three members of the research team. As recommended by Levac et $a l^{8}$ the research team will meet at the start, middle and end of the abstract review process to discuss any challenges and, if needed, to refine the search strategy. If any disagreement appears, a fourth research member will be consulted. Eligible articles will be retrieved in full-text and imported into EndNote to identify and discard duplicates. A flow diagram of the study search and selection process is presented in figure 1 .

\section{Stage 4: charting the data}

The full-text articles will be screened independently by three members of the research team using a charting form. The charting form has been developed to extract the study characteristic and findings, which will be piloted by two researchers on three articles and cross-checked for reliability. The charting form will include the inclusion criteria and an explanation of why the study is included or excluded at this stage in the process. Charting the data is also an iterative process, whereby the charting table will be continually updated. If there are any disagreements, a fourth researcher will be consulted until consensus is reached. Any changes will be highlighted in the final manuscript. Information that will be extracted includes study characteristics, designs and findings in relation

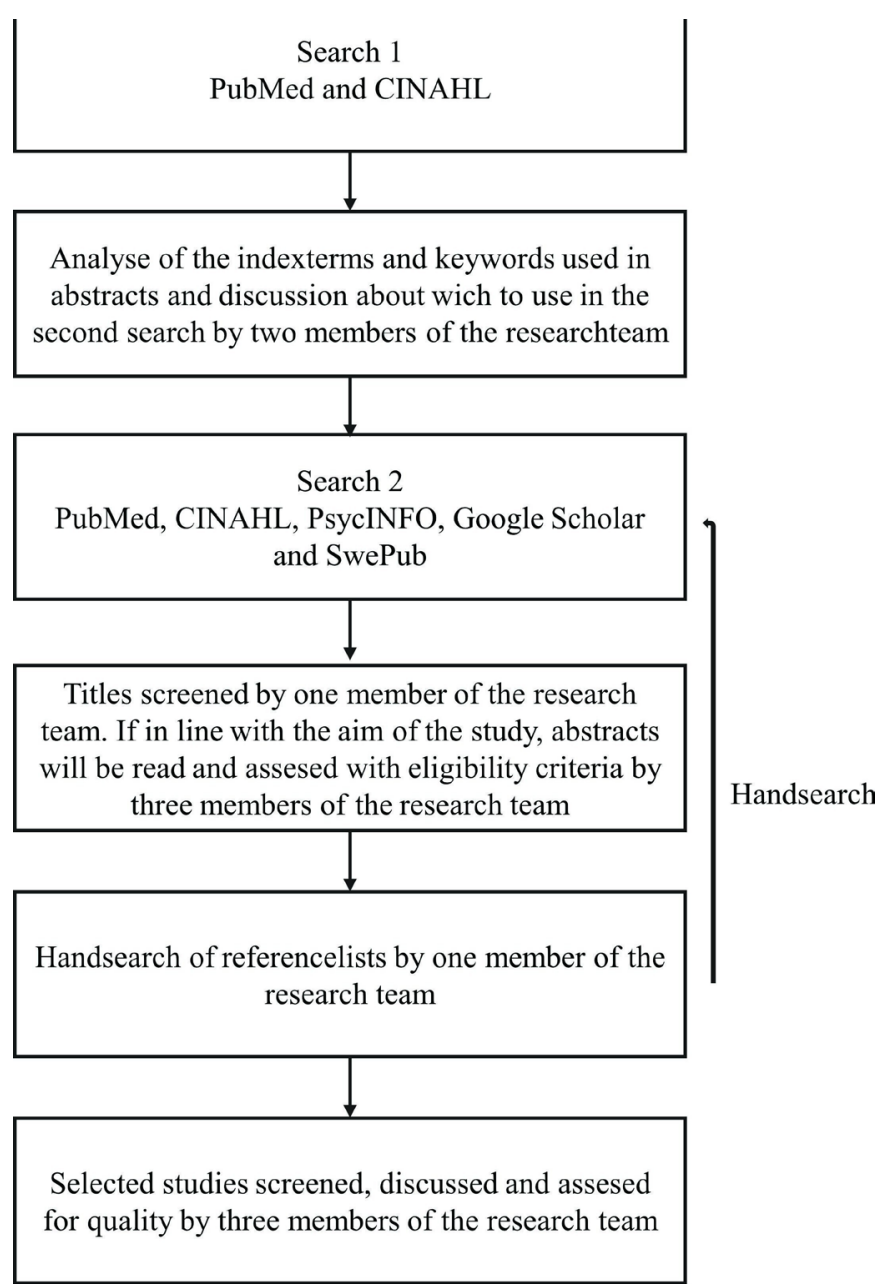

Figure 1 A flow diagram of the study search and selection process.

to the review question. The following findings will be extracted from the included studies:

(1) Model of support.

(2) Phase of the trajectory.

(3) Aim of the support.

(4) Participating family members.

Data that intend to be charted concerning model of support are: type of support, delivery mode and who delivers the support. Phase of trajectory relates to the timing of the support provided, for example, at diagnosis or during treatment. This might be modified during the process of charting the data.

In general, the quality of a study is not assessed in scoping reviews. ${ }^{9}$ However, the eligible full-text articles will be assessed using the Joanna Briggs Institution Critical Appraisal Tools ${ }^{13}$ as they enable a systematic exclusion of articles with incomplete methodological description. Thus, a cut-off will be set at studies not presenting: aim, criterions for inclusion and exclusion, participants, data collection, description and analyse of dropouts and the process of analysis. These studies will be categorised as having 'low quality'. Studies not selected for inclusion will be documented with reason for exclusion in a separate file. A visual flow diagram (PRISMA) ${ }^{14}$ will outline the 
decision-making process to enable replications by others and to further increase reliability of the findings and for methodological accuracy. ${ }^{9}$

\section{Stage 5: collating, summarising and reporting the results}

To support rigour, the reporting will be compliant with the PRISMA-ScR 22-item checklist. ${ }^{12}$ In addition, a descriptive summary of the scoping review process and of study characteristics will be presented. Subsequently, data will be analysed using inductive methodology and thematic analysis presented by Braun and Clarke..$^{15}$ Thematic analysis is a method for identifying, analysing and reporting patterns within data. It allows a large amount of data and can highlight differences and similarities across a data set. This stage will be segmented into the three steps suggested by Levac et al : analysing the data; reporting the results linked to the research question; and interpreting the implications of the results for research policy and practice. Lastly, a descriptive (narrative) summary of the included articles and the results that relate to the research question will be presented.

\section{Stage 6: consultation}

Even though consultation is optional, ${ }^{9}$ it enhances the methodological rigour and the validity of the outcome. Therefore, it is suggested as a compulsory stage in a scoping review. ${ }^{8}$ Thus, to confirm our findings, contact nurses at a cancer clinic will be identified and approached for consultation by a gatekeeper. Subsequently, the identified contact nurses will be informed about the purpose of the consultation and, if consenting, provided with the preliminary results. After a weeks' time to reflect about the findings, the participants will be contacted digitally at their convenience by the first author and asked to share their thoughts. According to Swedish Law, this consultation does not require ethical approval from the Swedish Ethical Review Authority.

\section{ETHICS AND DISSIMINATION}

The aim of this scoping review is to map the existing literature on support provided to family members during the continuum of cancer care. Since the methodology applied consists of reviewing and collecting data from publicly available material, this study does not require an ethical approval. To disseminate the findings an upcoming scoping review will be published in a scientific journal and presented at relevant conferences. In addition, the findings will be used to inform the design of a future study aiming to develop a model of support to family members during the trajectory of colorectal cancer.
Contributors MS led the design, search strategy and conceptualisation of this work and drafted the protocol. AW, JJ and MB were involved in the conceptualisation of the review design, inclusion and exclusion criteria and provided feedback on the methodology and the manuscript. All authors give their approval to the publishing of this protocol manuscript.

Funding The authors have not declared a specific grant for this research from any funding agency in the public, commercial or not-for-profit sectors.

Competing interests None declared.

Patient and public involvement Patients and/or the public were not involved in the design, or conduct, or reporting, or dissemination plans of this research.

Patient consent for publication Not required.

Provenance and peer review Not commissioned; externally peer reviewed.

Open access This is an open access article distributed in accordance with the Creative Commons Attribution Non Commercial (CC BY-NC 4.0) license, which permits others to distribute, remix, adapt, build upon this work non-commercially, and license their derivative works on different terms, provided the original work is properly cited, appropriate credit is given, any changes made indicated, and the use is non-commercial. See: http://creativecommons.org/licenses/by-nc/4.0/.

\section{ORCID iD}

Maria Samuelsson http://orcid.org/0000-0001-8700-4490

\section{REFERENCES}

1 Norlyk A, Martinsen B. The extended arm of health professionals? relatives' experiences of patient's recovery in a fast-track programme. J Adv Nurs 2013;69:1737-46.

2 den Bakker CM, Schaafsma FG, Huirne JAF, et al. Cancer survivors' needs during various treatment phases after multimodal treatment for colon cancer - is there a role for eHealth? BMC Cancer 2018;18:1207.

3 Aizer AA, Chen M-H, McCarthy EP, et al. Marital status and survival in patients with cancer. $J$ Clin Oncol 2013;31:3869-76.

4 Applebaum AJ, Breitbart W. Care for the cancer caregiver: a systematic review. Palliat Support Care 2013;11:231-52.

5 Möllerberg M-L, Sandgren A, Lithman T, et al. The effects of a cancer diagnosis on the health of a patient's partner: a populationbased registry study of cancer in Sweden. Eur J Cancer Care 2016;25:744-52.

6 Northouse L, Kershaw T, Mood D, et al. Effects of a family intervention on the quality of life of women with recurrent breast cancer and their family caregivers. Psychooncology 2005;14:478-91.

7 Coyne E, Wollin J, Creedy DK. Exploration of the family's role and strengths after a young woman is diagnosed with breast cancer: views of women and their families. Eur J Oncol Nurs 2012;16:124-30.

8 Levac D, Colquhoun H, O'Brien KK. Scoping studies: advancing the methodology. Implement Sci 2010;5:69.

9 Arksey H, O'Malley L. Scoping studies: towards a methodological framework. Int J Soc Res Methodol 2005;8:19-32.

10 Colquhoun HL, Levac D, O'Brien KK, et al. Scoping reviews: time for clarity in definition, methods, and reporting. $J$ Clin Epidemiol 2014:67:1291-4.

11 Institute JB. Joanna Briggs Institute Reviewers' Manual, 2015

12 Tricco AC, Lillie E, Zarin W, et al. PRISMA extension for scoping reviews (PRISMA-ScR): checklist and explanation. Ann Intern Med 2018;169:467-73.

13 Tufanaru CMZ, Aromataris E, Campbell J, et al. Chapter 3: systematic reviews of effectiveness. Joanna Briggs Institute Reviewer's Manual, 2017.

14 Moher D, Liberati A, Tetzlaff J, et al. Preferred reporting items for systematic reviews and meta-analyses: the PRISMA statement. PLoS Med 2009;6:e1000097.

15 Braun V, Clarke V. Using thematic analysis in psychology. Qual Res Psychol 2006;3:77-101. 